\title{
EXISTENCE OF NONOSCILLATORY SOLUTIONS OF FIRST ORDER NONLINEAR NEUTRAL EQUATIONS
}

\author{
LU WUDU ${ }^{1}$
}

(Received September 1989; revised February 1990)

\section{Abstract}

Consider the nonlinear neutral equation

$$
\left(x(t)-\sum_{i=1}^{m} p_{i}(t) x\left(h_{i}(t)\right)\right)^{\prime}+\sum_{j=1}^{n} f_{j}\left(t, x\left(g_{j}(t)\right)\right)=Q(t)
$$

where $p_{i}(t), h_{i}(t), g_{j}(t), Q(t) \in C\left[t_{0}, \infty\right), \lim _{t \rightarrow \infty} h_{i}(t)=\infty, \lim _{t \rightarrow \infty} g_{j}(t)=$ $\infty, i \in I_{m}=\{1,2, \ldots, m\}, j \in I_{n}=\{1,2, \ldots, n\}$. We obtain a necessary and sufficient condition (2) for this equation to have a nonoscillatory solution $x(t)$ with $\lim _{t \rightarrow \infty} \inf |x(t)|>0$ (Theorems 5 and 6 ) or to have a bounded nonoscillatory solution $x(t)$ with $\lim _{t \rightarrow \infty}$ inf $|x(t)|>0$ (Theorem 7).

\section{Introduction}

Consider the first order nonlinear neutral equation

$$
\left(x(t)-\sum_{i=1}^{m} p_{i}(t) x\left(h_{i}(t)\right)\right)^{\prime}+\sum_{j=1}^{n} f_{j}\left(t, x\left(g_{j}(t)\right)\right)=Q(t), \quad\left(t \geq t_{0}\right)
$$

where $p_{i}(t), h_{t}(t), g_{j}(t), Q(t) \in C\left[t_{0}, \infty\right), \lim _{t \rightarrow \infty} h_{i}(t)=\infty, \lim _{t \rightarrow \infty} g_{j}(t)$ $=\infty, i \in I_{m}=\{1,2, \ldots, m\}, j \in I_{n}=\{1,2, \ldots, n\}$.

Recently, many authors have studied oscillations of neutral equations. But since Grove et al [3] published the existence theorem of nonoscillatory solution of first order linear neutral equation with variable coefficients in 1987, there have been few results about the existence of nonoscillatory solutions, especially for nonlinear neutral equations.

\footnotetext{
${ }^{1}$ Department of Mathematics, South China Normal University, Guangzhou, 510631, China.

(C) Copyright Australian Mathematical Society 1990, Serial-fee code 0334-2700/90
} 
In this paper we study nonoscillations of (1) under very general conditions and obtain criterion theorems for the existence of nonoscillatory solutions of nonlinear neutral equations for the first time.

Neutral equations have applications in electric networks containing lossless transmission lines. Such networks arise in high speed computers where lossless transmission lines are used to interconnect switching circuits (see [1], [2], [4]).

A solution of (1) is called oscillatory if it has arbitrary large zeros and nonoscillatory if it is eventually positive or eventually negative.

\section{Main results}

We denote by $C_{B}$ all bounded continuous functions of $C\left[t_{0}, \infty\right)$. Define a distance in $C_{B}$ by

$$
R(x, y)=\sup _{t \geq t_{0}}|x(t)-y(t)|, \text { for } x, y \in C_{B} .
$$

Then $C_{B}$ becomes a complete metric space and every closed subset of $C_{B}$ is also a complete subspace.

We suppose that $f_{j}(t, x), j \in I_{n}, Q(t)$ satisfy the following conditions:

(a) $\left|f_{j}(t, x)\right| \leq\left|f_{j}(t, y)\right|$ when $|x| \leq|y|$;

(b) for each closed interval $L=[c, d](0<c<d)$, there are positive functions $L_{j}(t)\left(j \in I_{n}\right)$ such that

$$
\left|f_{j}(t, x)-f_{j}(t, y)\right| \leq L_{j}(t)|x-y| \text { when } x, y \in L
$$

where $L_{j}(t) \in C\left[t_{0}, \infty\right)$ are generally dependent on $L$ and $\int^{\infty} L_{j}(t) d t<$ $\infty, j \in I_{n}$;

(c) $\int^{\infty}|Q(t)| d t<\infty$.

THEOREM 1. Assume that either

$$
\mathrm{A}(\mathrm{i}): p_{i}(t) \geq 0, \quad i \in I_{m}, \quad \sum_{i=1}^{m} p_{\imath}(t) \leq 1-r, 0<r<1, t \geq t_{0},
$$

or

$$
\mathrm{A}(\mathrm{ii}): p_{t}(t) \leq 0, \quad i \in I_{m}, \quad-\sum_{i=1}^{m} p_{i}(t) \leq 1-r, 0<r<1, t \geq t_{0},
$$

holds and that $f_{j}(t, x)\left(j \in I_{n}\right)$ and $Q(t)$ satisfy condition (a)-(c). If

$$
\sum_{j=1}^{n} \int^{\infty}\left|f_{j}(t, d)\right| d t<\infty \text { for some } d \neq 0
$$


then (1) has a bounded nonoscillatory solution $x(t)$ with $\lim _{t \rightarrow \infty} \inf |x(t)|$ $>0$.

Proof. Set $S=\left\{x(t) \in C\left[t_{0}, \infty\right): c \leq x(t) \leq|d|, t \geq t_{0}\right\}$ where $0<c<$ $r|d|$. It is easy to see that $S$ is a complete metric space. Define a mapping as follows

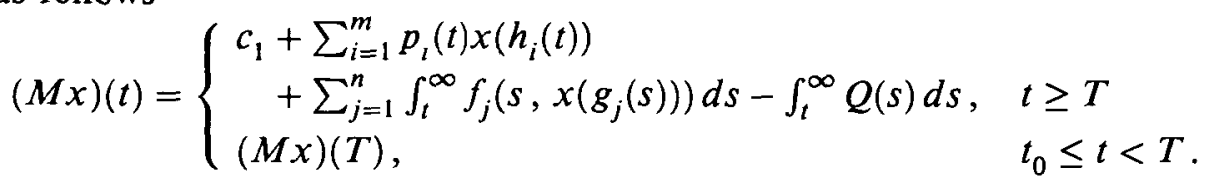

where $c_{1}$ and $T$ satisfy the following conditions:

When $\mathrm{A}(\mathrm{i})$ holds, $c<c_{1}<r|d|$ and $T$ is sufficiently large such that $h_{t}(t) \geq t_{0} \quad\left(i \in I_{m}\right)$ and $g_{j}(t) \geq t_{0} \quad\left(j \in I_{n}\right)$ as $t \geq T \geq t_{0}$ and further

$$
\begin{gathered}
\sum_{j=1}^{n} \int_{T}^{\infty}\left|f_{j}(s, d)\right| d s+\int_{T}^{\infty}|Q(s)| d s \leq \min \left\{c_{1}-c, r|d|-c_{1}\right\}, \\
\sum_{j=1}^{n} \int_{T}^{\infty} L_{j}(s) d s \leq \frac{r}{2} .
\end{gathered}
$$

When A(ii) holds, $c+(1-r)|d|<c_{1}<\frac{1}{2}(c+(2-r)|d|)$ and $T$ is sufficiently large such that $h_{l}(t) \geq t_{0}$ for $i \in I_{m}, g_{j}(t) \geq t_{0}$ for $j \in I_{n}$ as $t \geq T \geq t_{0}$, (4) holds and

$$
\sum_{j=1}^{n} \int_{T}^{\infty}\left|f_{j}(s, d)\right| d s+\int_{T}^{\infty}|Q(s)| d s \leq c_{1}-c-(1-r)|d| .
$$

We need to prove

(i) $M(S) \subset S$. If $\mathrm{A}(\mathrm{i})$ holds, for any $x \in S$ and by (3), we have when $t \geq T$

$$
\begin{aligned}
(M x)(t) & =c_{1}+\sum_{t=1}^{m} p_{i}(t) x\left(h_{i}(t)\right)+\sum_{j=1}^{n} \int_{t}^{\infty} f_{j}\left(x, s\left(g_{j}(s)\right)\right) d s-\int_{t}^{\infty} Q(s) d s \\
& \geq c_{1}-\sum_{j=1}^{n} \int_{t}^{\infty}\left|f_{j}\left(s, x\left(g_{j}(s)\right)\right)\right| d s-\int_{t}^{\infty}|Q(s)| d s \\
& \geq c_{1}-\sum_{j=1}^{n} \int_{T}^{\infty}\left|f_{j}(s, d)\right| d s-\int_{T}^{\infty}|Q(s)| d s \\
& \geq c_{1}-\left(c_{1}-c\right)=c \\
(M x)(t) & \leq c_{1}+|d| \sum_{i=1}^{m} p_{i}(t)+\sum_{j=1}^{n} \int_{T}^{\infty}\left|f_{j}(s, d)\right| d s+\int_{T}^{\infty}|Q(s)| d s \\
& \leq c_{1}+(1-r)|d|+\left(r|d|-c_{1}\right)=|d| .
\end{aligned}
$$


Since $(M x)(t)=(M x)(T)$ as $t_{0} \leq t<T$, then $c \leq(M x)(t) \leq|d|$ as $t_{0} \leq t<T$.

If A(ii) holds, by (5), we have when $t \geq T$

$$
\begin{aligned}
(M x)(t) & \geq c_{1}+|d| \sum_{i=1}^{m} p_{i}(t)-\sum_{j=1}^{n} \int_{T}^{\infty}\left|f_{j}(s, d)\right| d s-\int_{T}^{\infty}|Q(s)| d s \\
& \geq c_{1}-(1-r)|d|-\left(c_{1}-c-(1-r)|d|\right)=c, \\
(M x)(t) & \leq c_{1}+\sum_{j=1}^{n} \int_{T}^{\infty}\left|f_{j}(s, d)\right| d s+\int_{T}^{\infty}|Q(s)| d s \\
& \leq c_{1}+\left(c_{1}-c-(1-r)|d|\right) \leq|d| .
\end{aligned}
$$

Since $(M x)(t)=(M x)(T)$ as $t_{0} \leq t<T$, then $c \leq(M x)(t) \leq|d|$ as $t_{0} \leq t<T$.

(ii) $M$ is a compression mapping on $S$. For $x, y \in S$ and when $t \geq T$, we have

$$
\begin{aligned}
|(M x)(t)-(M y)(t)|= & \mid \sum_{i=1}^{m} p_{i}(t)\left(x\left(h_{i}(t)\right)-y\left(h_{i}(t)\right)\right) \\
& +\sum_{i=1}^{m} \int_{T}^{\infty}\left(f_{j}\left(s, x\left(g_{j}(s)\right)\right)-f_{j}\left(s, y\left(g_{j}(s)\right)\right)\right) d s \mid \\
\leq & \left(\sum_{i=1}^{m}\left|p_{i}(t)\right|\right) \sup _{t \geq t_{0}}|x(t)-y(t)| \\
& +\sum_{j=1}^{n} \int_{t}^{\infty} L_{j}(s)\left|x\left(g_{j}(s)\right)-y\left(g_{j}(s)\right)\right| d s \\
\leq & \left(\sum_{i=1}^{m}\left|p_{i}(t)\right|+\sum_{j=1}^{n} \int_{T}^{\infty} L_{j}(s) d s\right) R(x, y) \\
\leq & \left((1-r)+\frac{r}{2}\right) R(x, y)=\left(1-\frac{r}{2}\right) R(x, y)
\end{aligned}
$$

When $t_{0} \leq t<T$, we have

$$
|(M x)(t)-(M y)(t)|=|(M x)(T)-(M y)(T)| \leq\left(1-\frac{r}{2}\right) R(x, y)
$$

then

$$
R(M x, M y)=\sup _{t \geq t_{0}}|(M x)(t)-(M y)(t)| \leq\left(1-\frac{r}{2}\right) R(x, y) .
$$

According to the Banach fixed point theorem, $M$ has a fixed point $x^{*}$ in $S$. Obviously, $x^{*}(t)$ is a bounded nonoscillatory solution of $(1)$ and satisfies $\lim _{t \rightarrow \infty}$ inf $\left|x^{*}(t)\right|>0$. The proof is complete. 
THEOREM 2. Assume that either

$\mathrm{B}(\mathrm{i})$ : There is some integer $k(1 \leq k \leq m)$ such that $h_{k}(t)$ is strictly increasing, $h_{k}(t) \leq t, h_{i}(H(t)) \geq t(i \neq k), p_{k}(t)>1, p_{i}(t) \leq 0 \quad(i \neq k)$,

$$
\left|\frac{1}{p_{k}(t)}+\sum_{i \neq k} \frac{-p_{l}(t)}{p_{k}(t)}\right| \leq 1-r, \quad 0<r<1, t \geq t_{0}
$$

and when $t \geq t_{0}$ and $H\left(h_{i}(t)\right) \geq t_{0} \quad(i \neq k)$,

$$
\left|\frac{1}{p_{k}(H(t))}+\sum_{i \neq k} \frac{-p_{i}(t)}{p_{k}\left(H\left(h_{i}(t)\right)\right)}\right| \leq 1-r, \quad(0<r<1)
$$

where $H(t)$ is the inverse function of $h_{k}(t)$; or

$\mathrm{B}(\mathrm{ii})$ : There is some integer $k(1 \leq k \leq m)$ such that $h_{k}(t)$ is strictly increasing, $h_{k}(t) \leq t, h_{i}(H(t)) \geq t \quad(i \neq k), p_{k}(t)<-1, p_{i}(t) \leq 0 \quad(i \neq k)$ and (6) and (7) hold; is true. Further assume that $f_{j}(t, x)\left(j \in I_{n}\right)$ and $Q(t)$ satisfy condition (a)-(c). If (2) holds, then (1) has a bounded nonoscillatory solution $x(t)$, and when $p_{k}(t)$ is bounded, $\lim _{t \rightarrow \infty} \inf |x(t)|>0$.

Proof. If $\mathrm{B}(\mathrm{i})$ holds, set

$$
S=\left\{x(t) \in C\left[t_{0}, \infty\right): \begin{array}{ll}
\frac{c}{p_{k}(H(t))} \leq x(t) \leq \frac{|d|}{p_{k}(H(t))}, & t \geq T \\
\frac{c}{p_{k}(H(T))} \leq x(t) \leq \frac{|d|}{p_{k}(H(T))}, & t_{0} \leq t<T
\end{array}\right\}
$$

where $0<c<r|d|$. Choose a positive number $c_{1}$ such that $c<c_{1}<r|d|$. $T$ is sufficiently large such that $g_{j}(t) \geq t_{0},\left(j \in I_{n}\right)$ as $t \geq T \geq t_{0}$ and

$$
\begin{gathered}
\sum_{j=1}^{n} \int_{T}^{\infty}\left|f_{j}(s, d)\right| d s+\int_{T}^{\infty}|Q(s)| d s \leq \min \left\{c_{1}-c, r|d|-c_{1}\right\} \\
\sum_{j=1}^{n} \int_{T}^{\infty} L_{j}(s) d s \leq \frac{r}{2} .
\end{gathered}
$$

Define a mapping as follows:

$$
(M x)(t)=\left\{\begin{array}{l}
\frac{c_{1}}{p_{k}(H(t))}+\frac{x(H(t))}{p_{k}(H(t))}+\sum_{i \neq k} \frac{-p_{i}(H(t))}{p_{k}(H(t))} x\left(h_{i}(H(t))\right) \\
\quad+\frac{-1}{p_{k}(H(t))} \sum_{j=1}^{n} \int_{H(t)}^{\infty} f_{j}\left(s, x\left(g_{j}(s)\right)\right) d s \\
+\frac{1}{p_{k}(H(t))} \int_{H(t)^{Q(s) d s}, \quad}^{\infty} \quad t \geq T, \\
(M x)(T), \quad t_{0} \leq t<T .
\end{array}\right.
$$


We need to prove

(i) $M(S) \subset S$. For any $x \in S$ and from (7), (8), and (9), we have when $t \geq T$,

$$
\begin{aligned}
(M x)(t) & \geq \frac{1}{p_{k}(H(t))}\left(c_{1}-\sum_{j=1}^{n} \int_{H(t)}^{\infty}\left|f_{j}\left(s, x\left(g_{j}(s)\right)\right)\right| d s-\int_{H(t)}^{\infty}|Q(s)| d s\right) \\
& \geq \frac{1}{p_{k}(H(t))}\left(c_{1}-\sum_{j=1}^{n} \int_{T}^{\infty}\left|f_{j}(s, d)\right| d s-\int_{T}^{\infty}|Q(s)| d s\right) \\
& \geq \frac{1}{p_{k}(H(t))}\left(c_{1}-\left(c_{1}-c\right)\right)=\frac{c}{p_{k}(H(t))}, \\
(M x)(t) & \leq \frac{1}{p_{k}(H(t))}\left(c_{1}+\frac{|d|}{p_{k}(H(H(t)))}+\sum_{i \neq k} \frac{-p_{i}(H(t))|d|}{p_{k}\left(H\left(h_{l}(H(t))\right)\right)}\right. \\
& \left.+\sum_{j=1}^{n} \int_{T}^{\infty}\left|f_{j}(s, d)\right| d s+\int_{T}^{\infty}|Q(s)| d s\right) \\
& \leq \frac{1}{p_{k}(H(t))}\left(c_{1}+(1-r)|d|+\left(r|d|-c_{1}\right)\right)=\frac{|d|}{p_{k}(H(t))} .
\end{aligned}
$$

Since $(M x)(t)=(M x)(T)$ as $t_{0} \leq t<T$, then

$$
\frac{c}{p_{k}(H(T))} \leq(M x)(t) \leq \frac{|d|}{p_{k}(H(T))} \text { when } t_{0} \leq t<T
$$

(ii) $M$ is a compression mapping on $S$. For $x, y \in S$ and from (6) and (9), we have when $t \geq T$,

$$
\begin{aligned}
|(M x)(t)-(M y)(t)|=\mid & \frac{x(H(t))-y(H(t))}{p_{k}(H(t))} \\
& +\sum_{i \neq k} \frac{-p_{t}(H(t))}{p_{k}(H(t))}\left(x\left(h_{i}(H(t))\right)-y\left(h_{i}(H(t))\right)\right) \\
& -\frac{1}{p_{k}(H(t))} \sum_{j=1}^{n} \int_{H(t)}^{\infty}\left(f_{j}\left(s, x\left(g_{j}(s)\right)\right)\right. \\
& \left.-f_{j}\left(s, y\left(g_{j}(s)\right)\right)\right) d s \mid
\end{aligned}
$$

(continues) 


$$
\begin{aligned}
& \leq\left|\frac{1}{p_{k}(H(t))}+\sum_{i \neq k} \frac{-p_{i}(H(t))}{p_{k}(H(t))}\right| R(x, y) \\
& \quad+\sum_{j=1}^{n} \int_{H(t)}^{\infty} L_{j}(s)\left|x\left(g_{j}(s)\right)-y\left(g_{j}(s)\right)\right| d s \\
& \leq(1-r) R(x, y)+\left(\sum_{j=1}^{n} \int_{T}^{\infty} L_{j}(s) d s\right) R(x, y) \\
& \leq((1-r)+r / 2) R(x, y)=(1-r / 2) R(x, y)
\end{aligned}
$$

When $t_{0} \leq t<T$, we have

$$
|(M x)(t)-(M y)(t)|=|(M x)(T)-(M y)(T)| \leq(1-r / 2) R(x, y)
$$

Hence $R(M x, M y) \leq(1-r / 2) R(x, y)$. According to the Banach fixed point theorem, $M$ has a fixed $x^{*}$ in $S$. Obviously, when $t$ is sufficiently large, $x^{*}(t)$ satisfies

$$
\begin{aligned}
x^{*}\left(h_{k}(t)\right)= & \frac{c_{1}}{p_{k}(t)}+\frac{x^{*}(t)}{p_{k}(t)}+\sum_{i \neq k} \frac{-p_{i}(t)}{p_{k}(t)} x^{*}\left(h_{i}(t)\right) \\
& -\frac{1}{p_{k}(t)} \sum_{j=1}^{n} \int_{t}^{\infty} f_{j}\left(s, x^{*}\left(g_{j}(s)\right)\right) d s+\frac{1}{p_{k}(t)} \int_{t}^{\infty} Q(s) d s
\end{aligned}
$$

and then

$$
\left(x^{*}(t)-\sum_{i=1}^{m} p_{i}(t) x^{*}\left(h_{i}(t)\right)\right)^{\prime}+\sum_{j=1}^{n} f_{j}\left(t, x^{*}\left(g_{j}(t)\right)\right)=Q(t) .
$$

Hence $x^{*}(t)$ is a bounded nonoscillatory solution of (1) and when $p_{k}(t)$ is bounded, $\lim _{t \rightarrow \infty} \inf \left|x^{*}(t)\right|>0$. If $B($ ii) holds, set

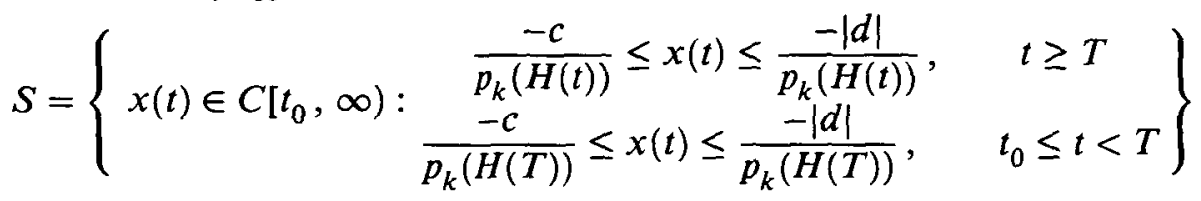

where $0<c<r|d|$. Choose a positive number $c_{1}$ such that $c+(1-r)|d|<$ $c_{1}<r(c+(2-r)|d|) / 2$. $T$ is sufficiently large such that when $t \geq T \geq t_{0}$, $g_{j}(t) \geq t_{0}\left(j \in I_{n}\right)$ and

$$
\begin{gathered}
\sum_{j=1}^{n} \int_{T}^{\infty}\left|f_{j}(s, d)\right| d s+\int_{T}^{\infty}|Q(s)| d s \leq c_{1}-c-(1-r)|d| \\
\sum_{j=1}^{n} \int_{T}^{\infty} L_{j}(s) d s \leq \frac{r}{2}
\end{gathered}
$$


Define a mapping as follows:

$$
(M x)(t)=\left\{\begin{aligned}
\frac{-c_{1}}{p_{k}(H(t))} & +\frac{x(H(t))}{p_{k}(H(t))}+\sum_{i \neq k} \frac{-p_{i}(H(t))}{p_{k}(H(t))} x\left(h_{l}(H(t))\right) \\
& +\frac{-1}{p_{k}(H(t))} \sum_{j=1}^{n} \int_{H(t)}^{\infty} f_{j}\left(s, x\left(g_{j}(s)\right)\right) d s \\
& +\frac{1}{p_{k}(H(t))} \int_{H(t)}^{\infty} Q(s) d s, \quad t \geq T \\
(M x)(T), & t_{0} \leq t<T .
\end{aligned}\right.
$$

Using (6), (7), (10), and (11) and by a proof similar to that of $B(i)$, we can complete our proof.

REMARK 1. If $p_{k}(t)$ is a constant, it is easy to see that $(6)$ is identical with (7) and Theorem 2 is still true when we omit the conditions $h_{k}(t) \leq t$ and $h_{i}(H(t)) \geq t \quad(i \neq k)$.

The following discussion is about the nonoscillation of $(1)$ when $p_{t}(t)$ are oscillatory functions.

TheOREM 3. Assume that

$$
C: \quad \sum_{i=1}^{m}\left|p_{i}(t)\right| \leq 1-r, \quad t \geq t_{0}, \quad\left(\frac{1}{2}<r<1\right)
$$

holds and $f_{j}(t, x)\left(j \in I_{n}\right), Q(t)$ satisfy conditions (a)-(c). If (2) holds, then (1) has a bounded nonoscillatory solution $x(t)$ with $\lim _{t \rightarrow \infty} \inf |x(t)|>0$.

Proof. Set $S=\left\{x(t) \in C\left[t_{0}, \infty\right): c \leq x(t) \leq|d|, t \geq t_{0}\right\}$ where $0<c<$ $(2 r-1)|d|$. Define a mapping as follows:

$$
(M x)(t)=\left\{\begin{array}{l}
c_{1}+\sum_{i=1}^{m} p_{i}(t) x\left(h_{t}(t)\right) \\
\quad+\sum_{j=1}^{n} \int_{t}^{\infty} f_{j}\left(s, x\left(g_{j}(s)\right)\right) d s-\int_{t}^{\infty} Q(s) d s, \quad t \geq T, \\
(M x)(T), \quad t_{0} \leq t<T ;
\end{array}\right.
$$

where $c_{1}$ satisfies $c+(1-r)|d|<c_{1}<r|d|$ and $T$ is sufficiently large such that when $t \geq T \geq t_{0}, h_{i}(t) \geq t_{0} \quad(i \neq k), g_{j}(t) \geq t_{0} \quad\left(j \in I_{n}\right)$ and

$$
\begin{gathered}
\sum_{j=1}^{n} \int_{T}^{\infty}\left|f_{j}(s, d)\right| s d+\int_{T}^{\infty}|Q(s)| d s \\
\leq \min \left\{c_{1}-c-(1-r)|d|, r|d|-c_{1}\right\}, \\
\sum_{j=1}^{n} \int_{T}^{\infty} L_{j}(s) d s \leq \frac{r}{2} .
\end{gathered}
$$

The rest of the proof is similar to that of Theorem 1 and 2 . 
THEOREM 4. Assume that

$\mathrm{D}$ : There is some integer $k \quad(1 \leq k \leq m)$ such that $h_{k}(t)$ is strictly increasing, $h_{k}(t) \leq t, h_{i}(H(t)) \geq t \quad(i \neq k),\left|p_{k}(t)\right|>1$,

$$
\left|\frac{1}{p_{k}(t)}\right|+\sum_{i \neq k}\left|\frac{p_{i}(t)}{p_{k}(t)}\right| \leq 1-r, \quad t \geq t_{0}, \quad \frac{1}{2}<r<1,
$$

and when $t \geq t_{0}$ and $H\left(h_{i}(t)\right) \geq t_{0}(i \neq k)$,

$$
\left|\frac{1}{p_{k}(H(t))}\right|+\sum_{i \neq k}\left|\frac{p_{i}(t)}{p_{k}\left(H\left(h_{l}(t)\right)\right)}\right| \leq 1-r, \quad\left(\frac{1}{2}<r<1\right),
$$

where $H(t)$ is the inverse function of $h_{k}(t)$;

holds and $f_{j}(t, x) \quad\left(j \in I_{n}\right)$ and $Q(t)$ satisfy (a)-(c). If (2) holds, then (1) has a bounded nonoscillatory solution $x(t)$, and when $p_{k}(t)$ is bounded, $\lim _{t \rightarrow \infty} \inf |x(t)|>0$.

Proof. Set

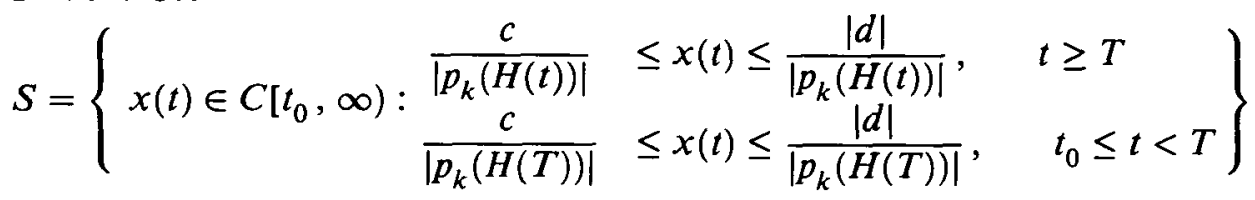
where $0<c<(2 r-1)|d|$. Choose $c_{1}$ such that $c+(1-r)|d|<c_{1}<r|d|$. $T$ is sufficiently large such that when $t \geq T \geq t_{0}, g_{j}(t) \geq t_{0} \quad\left(j \in I_{n}\right),(12)$ and (13) hold. Define a mapping as follows:

$$
(M x)(t)=\left\{\begin{aligned}
\frac{c_{1}}{\left|p_{k}(H(t))\right|} & +\frac{x(H(t))}{p_{k}(H(t))}+\sum_{i \neq k} \frac{-p_{i}(H(t))}{p_{k}(H(t))} x\left(h_{i}(H(t))\right) \\
& +\frac{-1}{p_{k}(H(t))} \sum_{j=1}^{n} \int_{H(t)}^{\infty} f_{j}\left(s, x\left(g_{j}(s)\right)\right) d s \\
& +\frac{1}{p_{k}(H(t))} \int_{H(t)}^{\infty} Q(s) d s, \quad t \geq T \\
(M x)(T), & t_{0} \leq t<T .
\end{aligned}\right.
$$

The rest of the proof is similar to that of Theorem 1 and 2 .

REMARK 2. If $p_{k}(t)$ is constant, (14) is identical with (15) and Theorem 4 is still true when we omit the conditions $h_{k}(t) \leq t$ and $h_{i}(H(t)) \geq t \quad(i \neq k)$. Consider the linear equation

$$
\left(x(t)-\sum_{i=1}^{m} p_{i}(t) x\left(h_{i}(t)\right)\right)^{\prime}+\sum_{j=1}^{n} q_{j}(t) x\left(g_{j}(t)\right)=Q(t), \quad t \geq t_{0}
$$

where $p_{t}(t), q_{j}(t), h_{i}(t), g_{j}(t), Q(t) \in C\left[t_{0}, \infty\right), \lim _{t \rightarrow \infty} h_{i}(t)=\infty \quad(i \epsilon$ $\left.I_{m}\right), \lim _{t \rightarrow \infty} g_{j}(t)=\infty \quad\left(j \in I_{n}\right)$. From Theorem 1-4, we have 
Corollary 1. Assume that one of A(i), A(ii), B(i), B(ii), C and D holds and that $p_{k}(t)$ of $\mathrm{B}(\mathrm{i}), \mathrm{B}(\mathrm{ii})$ and $\mathrm{D}$ is bounded. Further assume that $Q(t)$ satisfies (c). If

$$
\sum_{j=1}^{n} \int^{\infty}\left|q_{j}(s)\right| d s<\infty
$$

then (1)' has a bounded nonoscillatory solution $x(t)$ with $\lim _{t \rightarrow \infty} \inf |x(t)|$ $>0$.

Lemma 1. Assume that $\mathrm{A}(\mathrm{ii})$ or $\mathrm{B}(\mathrm{ii})$ holds and $Q(t)$ satisfies (c). Further assume that $f_{j}(t, x)\left(j \in I_{n}\right)$ satisfy (a) and

$$
x f_{j}(t, x) \geq 0 \quad(x \neq 0), j \in I_{n} .
$$

If (1) has a nonoscillatory solution $x(t)$ with $\lim _{t \rightarrow \infty}$ inf $|x(t)|>0$, then (2) holds.

Proof. Without loss of generality, assume that $x(t) \geq d>0 \quad\left(t \geq t_{0}\right)$. Set

$$
y(t)=x(t)-\sum_{i=1}^{m} p_{i}(t) x\left(h_{i}(t)\right) .
$$

If (2) does not hold, we have for $t \geq T$,

$$
y(t)-y(T) \leq \int_{T}^{t} Q(s) d s-\sum_{j=1}^{n} \int_{T}^{t} f_{j}(s, d) d s \rightarrow-\infty \quad(t \rightarrow \infty) .
$$

Then $\lim _{t \rightarrow \infty} y(t)=-\infty$. This is a contradiction. The proof is complete.

Lemma 2. Assume that $\mathrm{A}(\mathrm{i})$ or $\mathrm{C}$ holds, $h_{t}(t) \leq t \quad\left(i \in I_{m}\right)$ and that $Q(t)$ satisfies $(\mathrm{c})$ and $f_{j}(t, x) \quad\left(j \in I_{n}\right)$ satisfy (a) and (d). If (1) has a nonoscillatory solution $x(t)$ with $\lim _{t \rightarrow \infty} \inf |x(t)|>0$, then (2) holds.

Proof. Without loss of generality, assume that $x(t) \geq d>0 \quad\left(t \geq t_{0}\right)$. If (2) doesn't hold, we have $\lim _{t \rightarrow \infty} y(t)=-\infty$. Then $x(t)$ is unbounded. There is a sequence $t_{k} \rightarrow \infty \quad(k \rightarrow \infty)$ such that $x\left(t_{k}\right)=\max _{t \leq t_{k}} x(t)$. Then

$$
y\left(t_{k}\right)=x\left(t_{k}\right)-\sum_{i=1}^{m} p_{i}\left(t_{k}\right) x\left(h_{i}\left(t_{k}\right)\right) \geq\left(1-\sum_{i=1}^{m}\left|p_{i}\left(t_{k}\right)\right|\right) x\left(t_{k}\right)>0
$$

which is a contradiction. The proof is complete.

The following lemma is obvious. We omit the proof. 
Lemma 3. Assume that one of $\mathrm{A}(\mathrm{i}), \mathrm{A}(\mathrm{ii}), \mathrm{B}(\mathrm{i}), \mathrm{B}(\mathrm{ii}), \mathrm{C}$ and $\mathrm{D}$ holds and that $p_{k}(t)$ of $\mathrm{B}(\mathrm{i}), \mathrm{B}(\mathrm{ii})$ and $\mathrm{D}$ is bounded. Further assume that $Q(t)$ satisfies (c) and $f_{j}(t, x)\left(j \in I_{n}\right)$ satisfy (a) and (d). If (1) has a bounded nonoscillatory solution $x(t)$ with $\lim _{t \rightarrow \infty}$ inf $|x(t)|>0$, then (2) holds.

From Lemma 1-3 and Theorem 1-4, we have immediately

TheOREM 5. Assume that $\mathrm{A}(\mathrm{ii})$ or $\mathrm{B}(\mathrm{ii})$ holds and that $p_{k}(t)$ of $\mathrm{B}(\mathrm{ii})$ is bounded. Further assume that $Q(t)$ satisfies (c) and $f_{j}(t, x)\left(j \in I_{n}\right)$ satisfy (a), (b) and (d). Then (2) is a necessary and sufficient condition for (1) to have a nonoscillatory solution $x(t)$ with $\lim _{t \rightarrow \infty}$ inf $|x(t)|>0$.

THEOREM 6. Assume that $\mathrm{A}(\mathrm{i})$ or $\mathrm{C}$ holds and $h_{i}(t) \leq t \quad\left(i \in I_{m}\right)$. Further assume that $Q(t)$ satisfies (c) and $f_{j}(t, x) \quad\left(j \in I_{n}\right)$ satisfy (a), (b) and (d). Then (2) is a necessary and sufficient condition for (1) to have a nonoscillatory solution $x(t)$ with $\lim _{t \rightarrow \infty} \inf |x(t)|>0$.

TheOREM 7. Assume that one of $\mathrm{A}(\mathrm{i}), \mathrm{A}(\mathrm{ii}), \mathrm{B}(\mathrm{i}), \mathrm{B}(\mathrm{ii}), \mathrm{C}$ and $\mathrm{D}$ holds and that $p_{k}(t)$ of $\mathrm{B}(\mathrm{i}), \mathrm{B}(\mathrm{ii})$ and $\mathrm{D}$ is bounded. Further assume that $Q(t)$ satisfies (c) and $f_{j}(t, x) \quad\left(j \in I_{n}\right)$ satisfy (a), (b) and (d). Then (2) is a necessary and sufficient condition for (1) to have a bounded nonoscillatory solution $x(t)$ with $\lim _{t \rightarrow \infty} \inf |x(t)|>0$.

Corollary 2. Let $q_{j}(t) \geq 0\left(j \in I_{n}\right)$. Assume that $\mathrm{A}$ (ii) or $\mathrm{B}$ (ii) holds and that $p_{k}(t)$ of $\mathrm{B}(\mathrm{ii})$ is bounded. Further assume that $Q(t)$ satisfies (c). Then $(2)^{\prime}$ is a necessary and sufficient condition for (1)' to have a nonoscillatory solution $x(t)$ with $\lim _{t \rightarrow \infty} \inf |x(t)|>0$.

Corollary 3. Let $q_{j}(t) \geq 0 \quad\left(j \in I_{n}\right)$. Assume that $\mathrm{A}(\mathrm{i})$ or $\mathrm{C}$ holds and $h_{i}(t) \leq t \quad\left(i \in I_{m}\right)$. Further assume that $Q(t)$ satisfies (c). Then (2) is a necessary and sufficient condition for $(1)^{\prime}$ to have a nonoscillatory solution $x(t)$ with $\lim _{t \rightarrow \infty}$ inf $|x(t)|>0$.

Corollary 4. Let $q_{j}(t) \geq 0\left(j \in I_{n}\right)$. Assume that one of $\mathrm{A}(\mathrm{i}), \mathrm{A}(\mathrm{ii}), \mathrm{B}(\mathrm{i})$, $\mathrm{B}(\mathrm{ii}), \mathrm{C}$ and $\mathrm{D}$ holds and that $p_{k}(t)$ of $\mathrm{B}(\mathrm{i}), \mathrm{B}(\mathrm{ii})$ and $\mathrm{D}$ is bounded. Further assume that $Q(t)$ satisfies (c). Then (2)' is a necessary and suffcient condition for $(1)^{\prime}$ to have a bounded nonoscillatory solution $x(t)$ with $\lim _{t \rightarrow \infty}$ inf $|x(t)|>0$. 


\section{Examples}

Example 1. Consider the equation

$$
\left(x(t) \pm \frac{3}{5} x\left(h_{1}(t)\right) \pm \frac{1}{6+t^{2}} x\left(h_{2}(t)\right)\right)^{\prime}+\frac{1}{t^{2}} x^{3}(g(t))=e^{-t} \quad\left(t \geq t_{0}>0\right)
$$

where $h_{i}(t) \quad(i=1,2), g(t) \in C\left[t_{0}, \infty\right), \lim _{t \rightarrow \infty} h_{i}(t)=\infty \quad(i=1,2)$, $\lim _{t \rightarrow \infty} g(t)=\infty$. Let $p_{1}(t)=\mp \frac{3}{5}, p_{2}(t)=\mp \frac{1}{6+t^{2}}, Q(t)=e^{-t}, f(t, x)=$ $\frac{x^{3}}{t^{2}}$. Then

$$
\begin{aligned}
& \mp\left(p_{1}(t)+p_{2}(t)\right) \leq \frac{4}{5}, \quad \int^{\infty}|Q(t)| d t<\infty \\
& |f(t, x)|=t^{-2}|x|^{3} \leq t^{-2}|y|^{3}=|f(t, y)| \quad \text { when }|x| \leq|y| .
\end{aligned}
$$

When $x, y \in L=[c, d](0<c<d)$,

$$
|f(t, x)-f(t, y)|=t^{-2}\left|x^{2}+x y+y^{2}\right||x-y| \leq 3 d^{2} t^{-2}|x-y| .
$$

Let $L(t)=3 d^{2} t^{-2}$, then $\int^{\infty} L(t) d t<\infty$, and

$$
\int^{\infty}|f(t, d)| d t=\int^{\infty}|d|^{3} t^{-2} d t<\infty, \quad(d \neq 0) .
$$

According to Theorem $1,(16)$ has a bounded nonoscillatory solution $x(t)$ with $\lim _{t \rightarrow \infty}$ inf $|x(t)|>0$.

EXAMPLE 2. Consider the equation

$$
\left(x(t)-3\left(1+t^{2}\right) x\left(t-r_{1}\right)+t^{2} x\left(t-r_{2}\right)\right)^{\prime}+t^{-2} x^{2}(g(t))=0, \quad t \geq t_{0}>0,
$$

where $g(t) \in C\left[t_{0}, \infty\right), \lim _{t \rightarrow \infty} g(t)=\infty, r_{1}>0, r_{1}>r_{2}$. Let $p_{1}(t)=$ $3\left(1+t^{2}\right)>1, p_{2}(t)=-t^{2}<0, h_{1}(t)=t-r_{1}<t, h_{2}(t)=t-r_{2}, H(t)=t+r_{1}$ is the inverse function of $h_{1}(t)$, then $h_{2}(H(t))=t+r_{1}-r_{2}>t$.

$$
\begin{aligned}
& \left|\frac{1}{p_{1}(t)}+\frac{-p_{2}(t)}{p_{1}(t)}\right|=\frac{1}{3} \\
& \left|\frac{1}{p_{1}(H(t))}+\frac{-p_{2}(t)}{p_{1}\left(H\left(h_{2}(t)\right)\right)}\right| \leq\left|\frac{1}{3\left(1+t^{2}\right)}+\frac{t^{2}}{3\left(1+t^{2}\right)}\right|=\frac{1}{3}, \quad t \geq t_{0}
\end{aligned}
$$

Let $f(t, x)=t^{-2} x^{2}, L=[c, d] \quad(0<c<d)$, then for $x, y \in L$,

$$
|f(t, x)-f(t, y)|=t^{-2}|x+y||x-y| \leq 2 d t^{-2}|x-y| .
$$

Set $L(t)=2 d t^{-2}$, then $\int^{\infty} L(t) d t<\infty$. It is easy to see that $f(t, x)$ also satisfies (a) and (2). According to Theorem 2, (17) has a bounded nonoscillatory solution. 
EXAmple 3. Consider the equation

$$
\left(x(t)+\frac{1}{3} \sin t x(h(t))\right)^{\prime}+\frac{\cos t}{1+t^{2}} x(g(t))=Q(t), \quad t \geq 0,
$$

where $h(t), g(t), Q(t) \in C[0, \infty), \lim _{t \rightarrow \infty} h(t)=\infty, \lim _{t \rightarrow \infty} g(t)=\infty$, and $Q(t)$ satisfies (c). According to Theorem $3,(18)$ has a bounded nonoscillatory solution $x(t)$ with $\lim _{t \rightarrow \infty}$ inf $|x(t)|>0$.

EXAmple 4. Consider the equation

$$
\left(x(t)-5 x\left(h_{1}(t)\right)+\cos t x\left(h_{2}(t)\right)\right)^{\prime}+\frac{\cos t}{1+t^{2}}|x(g(t))|^{\alpha}=0, \quad t \geq 0,
$$

where $h_{1}(t), h_{2}(t), g(t) \in C\left[t_{0}, \infty\right), h_{1}(t)$ is strictly increasing, $\lim _{t \rightarrow \infty} h_{i}(t)$ $=\infty \quad(i=1,2), \lim _{t \rightarrow \infty} g(t)=\infty, 0<\alpha<1$. According to Theorem 4 and Remark 2, (19) has a bounded nonoscillatory solution $x(t)$ with $\lim _{t \rightarrow \infty} \inf |x(t)|>0$.

\section{References}

[1] R. K. Brayton, "Nonlinear oscillations in a distributed network", Quart. Appl. Math. 24 (1967) 289-301.

[2] R. K. Brayton and R. A. Willoughby, "On the numerical integration of a symmetric system of difference-differential equations of neutral type", J. Math. Appl. 18 (1967) $182-189$.

[3] E. A. Grove, M. R. S. Kulenovic and G. Ladas, "Sufficient conditions for oscillation and nonoscillation of neutral equations", J. Differential Equations 68 (1987) 373-382.

[4] J. Hale, Theory of functional differential equations (Springer-Verlag, New York, 1977). 